Revue d'histoire de l'enfance « irrégulière »

Le Temps de l'histoire

2| 1999

Cent ans de répressions des violences à enfants

\title{
L'actualité bibliographique
}

\section{(2) OpenEdition \\ 1 Journals}

Édition électronique

URL : http://journals.openedition.org/rhei/40

DOI : $10.4000 /$ rhei. 40

ISBN : 978-2-7535-1639-7

ISSN : $1777-540 \mathrm{X}$

Éditeur

Presses universitaires de Rennes

Édition imprimée

Date de publication : 15 novembre 1999

Pagination : 217-221

ISSN : 1287-2431

Référence électronique

"L'actualité bibliographique », Revue d'histoire de l'enfance « irrégulière » [En ligne], 2 | 1999, mis en ligne le 01 juin 2007, consulté le 04 décembre 2020. URL : http://journals.openedition.org/rhei/40 ; DOI :

https://doi.org/10.4000/rhei.40

(c) PUR 


\section{L'actualité bibliographique}

Rubrique à part entière du Temps de l'Histoire "L'Actualité bibliographique " cherche à répertorier le maximun de livres et d'articles de langue française qui traitent de l'enfance "irrégulière " (délinquante, malheureuse, abandonnée, etc.) et de sa prise en charge. Malgré tout le soin que nous apportons à la collecte des informations certaines références peuvent nous échapper. N'hésitez pas à nous signaler ces oublis, les titres omis seront intégrés dans la bibliographie du numéro suivant.

Le comité de rédaction

\section{A) INSTRUMENTS DE TRAVAIL ET DE RECHERCHE, TRAVAUX STATISTIQUES}

ROBERT (Philippe), SBRICCOLI (Mario), "Jalon pour une histoire de la statistique judiciaire”, Déviance et société, 1998, vol. 2, pp. 107-111.

REINKE (Herbert), "Une bonne statistique pour la lutte contre la criminalité? Observations sur les origines de la statistique criminelle en Allemagne au XIXe et au début du XXe siècle”, Déviance et société, 1998, vol. 2, pp. 113-125.

ROUSSEAUX (Xavier), TIXHON (Axel), STEVENS (Frédéric), “Origine de la statistique en Belgique (1795-1835)”, Déviance et société, 1998, vol. 2, pp. 127-153.

AUBUSSON de CAVARLAY (Bruno), "De la statistique criminelle apparente à la statistique judiciaire cachée”, Déviance et société, 1998, vol. 2, pp. 155-160.

CHAUVAUD (Frédéric), "Du bon usage du cabier bleu et du cabier jaune : 
de la statistique morale à la statistique instrumentale. Jalons d'une réflexion critique sur les usages de la statistique judiciaire", Déviance et société, 1998, vol. 2, pp. 181-200.

\section{B) TRAVAUX CENTRÉS SUR LES ASPECTS THÉORIQUES ET GÉNÉRAUX DE LA RÉÉDUCATION ET DU TRAVAIL SOCIAL}

ARVEILLER (Jean), "Pédophilie et psychiatrie, repères historiques", Évolution psychiatrique, tome 63, $\mathrm{n}^{\circ} 1-2$, janvier-juin 1998, pp. 11-34.

BOURQUIN (Jacques), "La difficile émergence de la notion d'éducabilité du mineur délinquant", Trames, $n^{\circ}$ 4-5, 1998, pp. 131-142.

CHARTIER (Jean-Pierre), "Voyage au bout de la rééducation", Topique, $\mathrm{n}^{\circ} 65,1998$, pp. 43-56.

KALUSZYNSKI (Martine), "Les artisans de la loi. Espaces juridico-politiques en France sous la IIIe République”, Droit et société, n 40, 1998, pp. 535-562.

MUCCHIELLI (Laurent), "Clochards et sans-abri : actualité de l'œuvre d'Alexandre Vexliard”, Revue française de sociologie, janvier-mars 1998, XXXIX-1, pp. 105-138.

RENNEVILLE (Marc), Essai sur l'émergence d'un regard médical sur la criminalité en France (1785-1885), Thèse d'histoire, Paris 7-Denis Diderot, Villeneuve d'Ascq, Presses universitaires du Septentrion, 1997, 2 vol., $920 \mathrm{p}$.

SENON (Jean-Louis), "Histoire de la psychiatrie en milieu pénitentiaire de Pinel à la loi du 18 janvier 1994", Annales médico-psychologiques, vol. $156, \mathrm{n}^{\circ} 3$, mai 1998 , pp. 161-178.

VIMONT (Jean-Claude), "Le docteur Vingtrinier et les mineurs de justice”, Trames, n4-5, 1998, pp. 157-167. 


\section{C) TRAVAUX CENTRÉS SUR LES “JEUNES"}

AMBROISE-RENDU (Anne-Claude), "La famille en morceaux. Représentation des violences familiales dans la chronique des faits divers, 1870-1910", Sociétés et représentations, nº 6, juin 1998, pp. 17-35.

BOURQUIN (Jacques), "On les appelait en 1950 les cas résiduels. Ils furent plus tard les incasables. La protection judiciaire et les mineurs difficiles”, Empan, n 32, décembre 1998, pp. 27-33.

CARON (Jean-Claude), "Les marques du savoir", Sociétés et représentations, $\mathrm{n}^{\circ}$ 6, juin 98, pp. 117-130.

DUPONT-BOUCHAT (Marie-Sylvie), "L'enfance violentée", Sociétés et représentations, $\mathrm{n}^{\circ}$ 6, juin 1998, pp. 153-178.

FARCY (Jean-Claude), "Quelques problèmes d'analyse de la délinquance juvénile à la fin du XIXe siècle. L'exemple parisien”, Trames, n ${ }^{\circ}$-5, 1998, pp. 143-156.

MICHAUD (Marie-Christine), "La rébellion des jeunes italo-américains au début du siècle. L'exemple de deux romans italo-américains”, Migrations société, vol. 10, n 58-59, juillet-octobre 1998, pp. 7-19.

KIENER (Michel C.), "Enfance et violences dans la France de 1880 à 1900", dans D'Hollander (Paul), Violences en Limousin à travers les siècles, Limoges, PULIM, 1998, pp. 197-234.

QUINCY-LEFEBVRE (Pascale), Familles, institutions et déviances. Histoire de l'enfance difficile : 1880-fin des années trente, Paris, Economica, 1997, $433 \mathrm{p}$.

TÉTARD (Françoise), "Les inéducables comme enjeu des politiques correctives depuis le XIXe siècle", Mascaret, revue régionale du CREAHI d'Aquitaine, $\mathrm{n}^{\circ}$ 51-52, 1er semestre 1998, pp. 35-46.

THIERCÉ (Agnès), "Pacifier l'adolescence, les choix des années 1890"; dans Adolescence, 1998, Tome 16, n 1, pp. 257-268.

TOURNERIE (Jean-André), Criminels et vagabonds au siècle des Lumières, Paris, Imago, 1997, $250 \mathrm{p}$.

VASSIGH (Denis Darya), "L'action juridique en faveur des enfants maltraités dans la deuxième moitié du XIXe siècle", Trames, n 4-5, 1998, pp. 169-179. 


\section{D) TRAVAUX CENTRÉS SUR LES PROFESSIONNELS}

BOUQUET (Brigitte), "Féminin-masculin chez les assistant(e)s de service social", Vie sociale, n 3, 1998, pp. 17-36

GARDET (Mathias), TÉTARD (Françoise), "Chercher les femmes! Femmes d'éducateurs et éducatrices”, Vie sociale, n 3, 1998, pp. 37-51. KNIBIEHLER (Yvonne), "Féminisme et travail social", Vie sociale, n 3 , 1998, pp. 9-15.

TÉTARD (Françoise), GARDET (Mathias), "Chercher les femmes! Femmes d'éducateurs et éducatrices”, Vie sociale, n 3, 1998, pp. 37-51.

\section{E) TRAVAUX CENTRÉS SUR LES INSTITUTIONS ET LES POLITIQUES PUBLIQUES}

BASSON (Jean-Charles), "Faut-il réprimer les violences scolaires? À propos du centenaire de la loi du 19 avril 1998", Cabiers de la sécurité intérieure, $\mathrm{n}^{\circ} 32,1998$, pp. 175-187.

BEC (Colette), L'assistance en démocratie. La politique assistancielle dans la France des XIXe et XXe siècles, Paris, Belin, 1998, 256 p.

CASTEL (Robert), "Du travail social à la gestion du non social”, Esprit, mars-avril, 1998, pp. 28-45.

CRAPUCHET (Simone), "Service, action et politique sociales d'outremer (1943-1960)”, Vie sociale, n 2, 1998, pp. 19-64.

DONZELOT (Jacques), ROMAN (Joël), "Les nouvelles données du social : 1972-1998”, Esprit, mars-avril, 1998, pp. 7-25.

FECTEAU Jean-Marie (et al.), "Une politique de l'enfance délinquante et en danger. La mise en place des écoles de réforme et d'industrie au Québec (1840-1873)”, Crime, histoire \& sociétés, $\mathrm{n}^{\circ}$ 1, vol. 2, 1998, pp. 75-110.

LARMINAT (Valérie), "Éducation scolaire - éducation spécialisée : histoire d'un contencieux", Actualités sociales hebdomadaires, n 2075, 12 juin 1998, pp.19-20.

PLANTET (Joël), “Comment les éducateurs ont perdu l'ANEJI", Lien 
social, n 449, 9 juillet 1998, pp. 4-6.

ROMAN (Joël), DONZELOT (Jacques), "Les nouvelles données du social : 1972-1998”, Esprit, mars-avril, 1998, pp. 7-25.

\section{F) SOUVENIRS, MÉMOIRES ET AUTOBIOGRAPHIES (PROFESSIONNELS ET 'JEUNES”)}

BLUM (Françoise), "Mémoires d'une assistante sociale coloniale. Entretien avec Simone Crapuchet", Vie sociale, n 2, 1998, pp. 5-15.

Drôle d'oiseau, Autobiographie d'un voyou de la Belle Époque, postface de Philippe Artières, Paris, Imago, 1998, 160 p.

LÉGER (Raoul), La colonie agricole et pénitentiaire de Mettray. Souvenirs d'un colon, suivi de contributions historiques de Jacques BOURQUIN et Éric PIERRE, Paris, L'Harmattan, 1998, 170 p.

PLANTET (Joël), "Mémoire d'un pionnier. Entretien avec Jacques Guyaumarc'h”, Lien social, n 449, 9 juillet 1998, pp. 7-9. 